\title{
The SkyMapper Transient Survey
}

\author{
R. A. Scalzo ${ }^{1,2,3},{ }^{*}$ F. Yuan ${ }^{1,2}$, M. J. Childress ${ }^{1,2,4}$, A. Möller ${ }^{1,2}$, B. P. Schmidt ${ }^{1,2}$, B. E. Tucker ${ }^{1,2}$, B. R. Zhang ${ }^{1,2}$, \\ P. Astier ${ }^{2,3}$, M. Betoule ${ }^{2,3}$, and N. Regnault ${ }^{2,3}$ \\ ${ }_{1}^{1}$ Research School of Astronomy and Astrophysics, Australian National University, Canberra, ACT 2611, Australia \\ 2 ARC Centre of Excellence for All-sky Astrophysics (CAASTRO) \\ ${ }^{3}$ Centre for Translational Data Science, University of Sydney, NSW 2006, Australia \\ ${ }_{5}^{4}$ School of Physics and Astronomy, University of Southampton, Southampton, SO17 1BJ, UK \\ ${ }^{5}$ Laboratoire de Physique Nucléaire et des Hautes Énergies, Université Pierre et Marie Curie Paris 6, Université Paris Diderot \\ Paris 7, CNRS-IN2P3, 4 place Jussieu, 75252 Paris Cedex 05, France
}

\begin{abstract}
The SkyMapper $1.3 \mathrm{~m}$ telescope at Siding Spring Observatory has now begun regular operations. Alongside the Southern Sky Survey, a comprehensive digital survey of the entire southern sky, SkyMapper will carry out a search for supernovae and other transients. The search strategy, covering a total footprint area of $\sim 2000 \mathrm{deg}^{2}$ with a cadence of $\leq 5$ days, is optimised for discovery and follow-up of low-redshift type Ia supernovae to constrain cosmic expansion and peculiar velocities. We describe the search operations and infrastructure, including a parallelised software pipeline to discover variable objects in difference imaging; simulations of the performance of the survey over its lifetime; public access to discovered transients; and some first results from the Science Verification data.
\end{abstract}

Keywords: (stars:) supernovae: general; (cosmology:) dark energy; methods: data analysis

\section{INTRODUCTION}

The advent of automated, wide-field survey telescopes has revolutionised astronomy by dramatically increasing the sky area that can be observed to a given depth in a short span of time. At the same time, automation and digitization of the end-to-end operation of these telescopes, from routine operation to data reduction to data storage, has produced an unprecedented wealth of data to mine for new patterns and objects. The surveys employing these telescopes have created digital maps of large sky areas, such as the Sloan Digital Sky Survey (SDSS; York et al. 2000). They also enable increasingly intensive, untargeted monitoring of large sky areas for variable and transient objects. Such monitoring reduces the selection bias associated with targeting particular sky areas or host galaxies, and results in large, homogeneous samples of all transient phenomena in the sky in the targeted wavelength range to a certain magnitude limit. Completed time-domain surveys with a footprint larger than $1000 \mathrm{deg}^{2}$ include Palomar-QUEST (Djorgovski et al. 2008) and the Palomar Transient Factory (PTF; Rau et al. 2009; Law et al. 2009). Ongoing wide-area time-domain surveys include Pan-STARRS (Kaiser et al. 2010), LaSilla-QUEST (Baltay et al. 2013), the iPTF extension to PTF, and the Catalina Real-Time Transient Survey (CRTS; Drake et al. 2009).

\footnotetext{
*rscalzo@anu.edu
}

The SkyMapper $1.3 \mathrm{~m}$ robotic telescope Keller et al. 2007) at Siding Spring Observatory has commenced the Southern Sky Survey, an automated, digital survey of the southern sky. Alongside this survey ("Main Survey"), the SkyMapper Transient Survey (SMT) is a search for supernovae and transients in the local Universe optimised to discover and follow up SNe Ia for cosmology.

A major science driver for time-domain surveys is the study of the Universe's accelerating expansion (Riess et al. 1998; Schmidt et al. 1998; Perlmutter et al. 1999) and the parameters of the "dark energy" which drives it, through the discovery and follow-up of type Ia supernovae (SNe Ia). Contemporary cosmological analyses such as the Joint Lightcurve Analysis (JLA; Betoule et al. 2014) require both high-redshift and low-redshift SNe Ia to make inferences about the dark energy equation of state; the lowredshift SNe Ia mainly constrain the mean absolute magnitude of $\mathrm{SNe} \mathrm{Ia}$, while the high-redshift $\mathrm{SNe}$ Ia use luminosity distances to map the Universe's scale factor over cosmic time. Presently the high-redshift SNe Ia sample is composed of magnitude-limited surveys such as the Supernova Legacy Survey (SNLS; Sullivan et al. 2011; Conley et al. 2011) and the ongoing Dark Energy Survey (DES; Dark Energy Survey Collaboration et al. 2016). Meanwhile the nearby supernova sample comes from a myriad of surveys through the 2000's such as the Harvard-Smithsonian Center for Astrophysics (CfA) surveys 
(CfA1-4; Riess et al. 1999; Jha et al. 2006; Hicken et al. 2009, 2012) and the Carnegie Supernova Project (CSP; Contreras et al. 2010), which follow up SNe discovered in the automated Lick Observatory Supernova Search (LOSS; Li et al. 2000; Filippenko et al. 2001) or by amateur astronomers. These supernovae commonly were found by targeting large nearby galaxies. The accuracy and precision with which cosmological parameters can currently be measured from SNe Ia are limited by systematic errors, particularly photometric calibration (Betoule et al. 2013, 2014), but also including uncertainties in dust extinction (Phillips et al. 2013; Scolnic et al. 2014; Burns et al. 2014), potential population diversity (Quimby et al. 2007; Wang et al. 2009; Kelly et al. 2010; Sullivan et al. 2010; Childress et al. 2013; Kelly et al. 2015), evolution (Kim et al. 2004; Howell et al. 2007; Sullivan et al. 2009; Milne et al. 2015) over a range of redshifts, or the influence of peculiar velocities (Davis et al. 2011) including coherent bulk flows (Hui \& Greene 2006).

Of the aforementioned systematics, the contributions to the uncertainty budget from photometric calibration and peculiar velocities are magnified by the heterogeneity of the low-redshift SN Ia sample, in particular by the numerous telescopes used to observe them, and by their nonuniform spatial distribution. SkyMapper aims to address these limitations by searching a wide sky area uniformly with a short ( $\leq 5$ days) cadence, in multiple well-determined bandpasses (Bessell et al. 2011). Thus the resultant lowredshift $(z<0.1)$ SN Ia sample will be well-calibrated and magnitude-limited, with a more similar selection function to the high-z sample. SMT will therefore be very useful for measurements of cosmic expansion and peculiar velocities associated with bulk flows and cosmic structure, and for studies of type Ia supernova physics aimed at improving $\mathrm{SNe}$ Ia as distance indicators. SkyMapper is also unique in its spatial overlap with the DES footprint, positioning SMT as an optimal low-redshift anchor.

Here we introduce the infrastructure and operations of the SkyMapper Transient Survey, and present some first results including performance during an early Science Verification period. The SN Survey has begun operating at scale from April 2015, and has released candidates and classifications to the public. This paper will be followed shortly by an early data release of $\sim 30 \mathrm{SNe}$ Ia to date, and by individual papers on peculiar transients such as superluminous supernovae (6.2). The structure of the paper is as follows: $\S 2$ gives an overview of SkyMapper telescope and the Main Survey. $\S 3$ describes the search pipeline and follow-up procedure, while $\S 4$ focuses on survey strategy. We discuss and evaluate the performance thus far in $\S 5$, and present some early results in $\S 6$.

\section{THE SKYMAPPER TELESCOPE AND MAIN SURVEY}

The SkyMapper telescope, and the Main Survey infrastructure and science goals, are described in detail in Keller et al.
(2007); we briefly summarise the most relevant details below.

SkyMapper is a $1.3 \mathrm{~m}, f / 4.8$ telescope at Siding Spring Observatory, operated by the Australian National University on behalf of the Australian astronomical community. The telescope has a $5.7 \mathrm{deg}^{2}$ field of view, covering a square $2.4 \mathrm{deg} \times 2.4 \mathrm{deg}$ area with a fill factor of $91 \%$. The 268-Mpix imager has a pixel scale of $0.5 \mathrm{arcsec} / \mathrm{pix}$. Available filters include SDSS-like griz, Stromgren $u$, and a custom-made, intermediate-band $v$ filter specific to SkyMapper (Bessell et al. 2011). The $v$ filter covers the range 3670$3980 \AA$, to allow simultaneous measurements of surface gravity and metallicity from broad-band photometry; it is optimised to enable the Main Survey's key science goals in galactic archaeology, particularly the identification of extremely metal-poor stars (e.g. Keller et al. 2014).

The calibration of the Southern Hemisphere standard stars in the SkyMapper photometric system is accomplished through a "Short Survey" of images with short (5-10 sec) exposure times, targeting secondary standard stars with magnitudes between 8.5 and 15.5 (Keller et al. 2007). This part of the survey requires at least three images of each part of the sky in all six SkyMapper filters, under photometric conditions. Photometric superflats are also formed by taking a series of dithered exposures, moving a standard star across the surface of the mosaic, to characterise and remove patterns of scattered light. The absolute zeropoint of SkyMapper will be determined from stars in the Walraven photometric system (Pel \& Lub 2007) with Hubble Space Telescope spectrophotometry from the Next Generation Spectral Library 1 Changes in the SkyMapper optical throughput will be monitored using a photodiode system Stubbs et al. 2007; Regnault et al. 2012).

SkyMapper's routine observing is fully automated. A schedule is input for each night as a list of observation requests, each comprising a desired bandpass, exposure time, and optimal window during which the observation can take place. Automated safety measures will close the dome in case of cloud or inclement weather. The Main Survey nightly schedules are determined each night according to a survey strategy which balances its various science goals, but also monitors the observing conditions (for example, seeing or photometricity) to trigger execution of third-party programs designed to take advantage of conditions unsuitable for Main Survey operations. The SN Survey is an example of such a third-party program. Observations can thus be scheduled in "classical" mode, occupying specific blocks of time, or in "queue" mode to respond to changing observing conditions.

\section{SKYMAPPER TRANSIENT SURVEY PIPELINE}

Although the SN Survey and the Main Survey both use the SkyMapper telescope, the SN Survey has its own data reduction pipeline infrastructure, maintained and run separately

\footnotetext{
${ }^{1}$ http://lifshitz.ucdavis.edu/ mgregg/gregg/ngsl/ngsl.html
} 
from the Main Survey data pipeline. This pipeline enables additional processing (e.g. image subtraction) beyond Main Survey requirements, ensures rapid $(<12 \mathrm{~h})$ turnaround for discovery of new transient candidates, and provides additional data to support situational awareness of active candidates, such as historical light curves, a web service enabling follow-up of transients, and annotations by users about the transient type and characteristics.

\subsection{Image subtraction workflow}

The image subtraction pipeline is written almost entirely in Python, with some $\mathrm{C}++$ extensions for pixel-level image processing (e.g. flat fielding) and incorporating commonly used open-source modules (e.g., Bertin \& Arnouts 1996) where available. The stages of a particular workflow are shown in Figure 1 Image subtraction for the SN Survey requires a pre-existing template image of the sky ("REF") to remove host galaxy light and non-variable sources from each new exposure ("NEW"). Image subtraction involves astrometric resampling and rescaling of the REF to match the NEW, and convolution of the REF by a suitably chosen kernel so that its point-spread function (PSF) matches that of the NEW. During Science Verification, a cache of REF images was built up automatically; now, if no REF image is available in a given part of the sky to subtract from a NEW image, the NEW image is simply added to the cache as a REF. We require that each REF image have a narrower PSF than the NEW image, so that the REF is always convolved to the NEW; this minimises correlations between pixels associated with newly discovered $\mathrm{SNe}$. We also require that the REF image be taken at least two weeks prior to the NEW image, since the rise time of a typical SN Ia to maximum light is about 17 days. Following the start of Main Survey operations in April 2015, the SN Survey began coordinating with the Main Survey to use completed Main Survey exposures as REF images for template subtraction. This ensures that suitably deep REF images exist in all four SN Survey filter bands (vgri) before beginning to search a given field, so that follow-up light curves can be generated immediately in any filter.

Reduction of a SkyMapper SN Survey exposure begins with a simplified version of the Main Survey pipeline workflow described in Keller et al. (2007). The mosaic images are split into individual $2048 \times 4096$ CCD images. An overscan region is subtracted from each half of the CCD (corresponding to different amplifiers). Flat fields are constructed nightly from dome flat images and applied to science exposures after overscan subtraction. A bad pixel map is created to flag pixels based on consistent deviation from a reasonable gain range and/or erratic behavior. A quick large-scale astrometric solution for the mosaic is produced using ASTROMETRY.NET. If the astrometric registration fails (e.g., because of heavy cloud), or if the image quality is poor (FWHM $>4$ arcsec, or elongation $>1.2$ ), the image is discarded at this stage and undergoes no further analysis. For each indi- vidual CCD of a NEW exposure, the workflow then proceeds through the following stages:

1. WCS: The world coordinate system for the NEW is refined, with higher-order distortions described in the zenithal polynomial $(\mathrm{ZPN})$ representation. This produces astrometry accurate to about 0.1 arcsec.

2. SExtractor: Sources are detected in the NEW image with SEXTRACTOR (Bertin \& Arnouts 1996) and aperture photometry is extracted over a series of apertures.

3. ZP: A preliminary photometric zeropoint is produced by comparison to APASS (Henden \& Munari 2014). Analysis of data from June 2014 suggests that the mean color terms for transforming between the APASS and SkyMapper gri filters are small $(<0.05)$. The SN Survey will eventually be tied to the same photometric system as the Main Survey, pending completion of Main Survey fields in the search area. If an image has no corresponding REF, it is added to the REF cache at this stage and no further processing takes place.

4. SWarp: The REF image is resampled to the coordinate system of the NEW image using SWARP (Bertin et al. 2002).

5. hotpants: The REF is scaled to the NEW flux level, convolved with a spatially-varying kernel to match the NEW PSF as accurately as possible, and subtracted from the NEW using HOTPANTS 2 to produce a subtracted image ("SUB"). Sources are detected in the SUB image with SEXTRACTOR. Each SUB image inherits the world coordinate system and photometric zeropoint of the corresponding NEW image.

6. classify: All detections on the SUB are run through an automated classification routine (see 3.3) to determine the likelihood that they are real astrophysical sources rather than artifacts from an imperfect subtraction process.

7. xref: All high-quality detections in the SUB image are astrometrically matched to previous detections. For sources passing a threshold number of high-quality detections in one or more subtractions, a historical light curve is compiled using all detections of the transient at that position.

Figure 2 shows the distribution of "wall times" for the processing of a single SkyMapper CCD (running on a single core). The median processing time is 115 seconds from initial reduction to automatic flagging of candidates. While this is slower than real-time, it is fast enough to process an entire night's worth of exposures in less than 24 hours, allowing the pipeline to keep up with the flow of data. During Science Verification, we found that the end-to-end success rate for subtraction jobs was close to $99 \%$, with a small number of failures easily traced to low-quality input data (due, for example, to poor weather conditions).

\footnotetext{
${ }^{2}$ http://www.astro.washington.edu/users/becker/v2.0/hotpants.html
} 


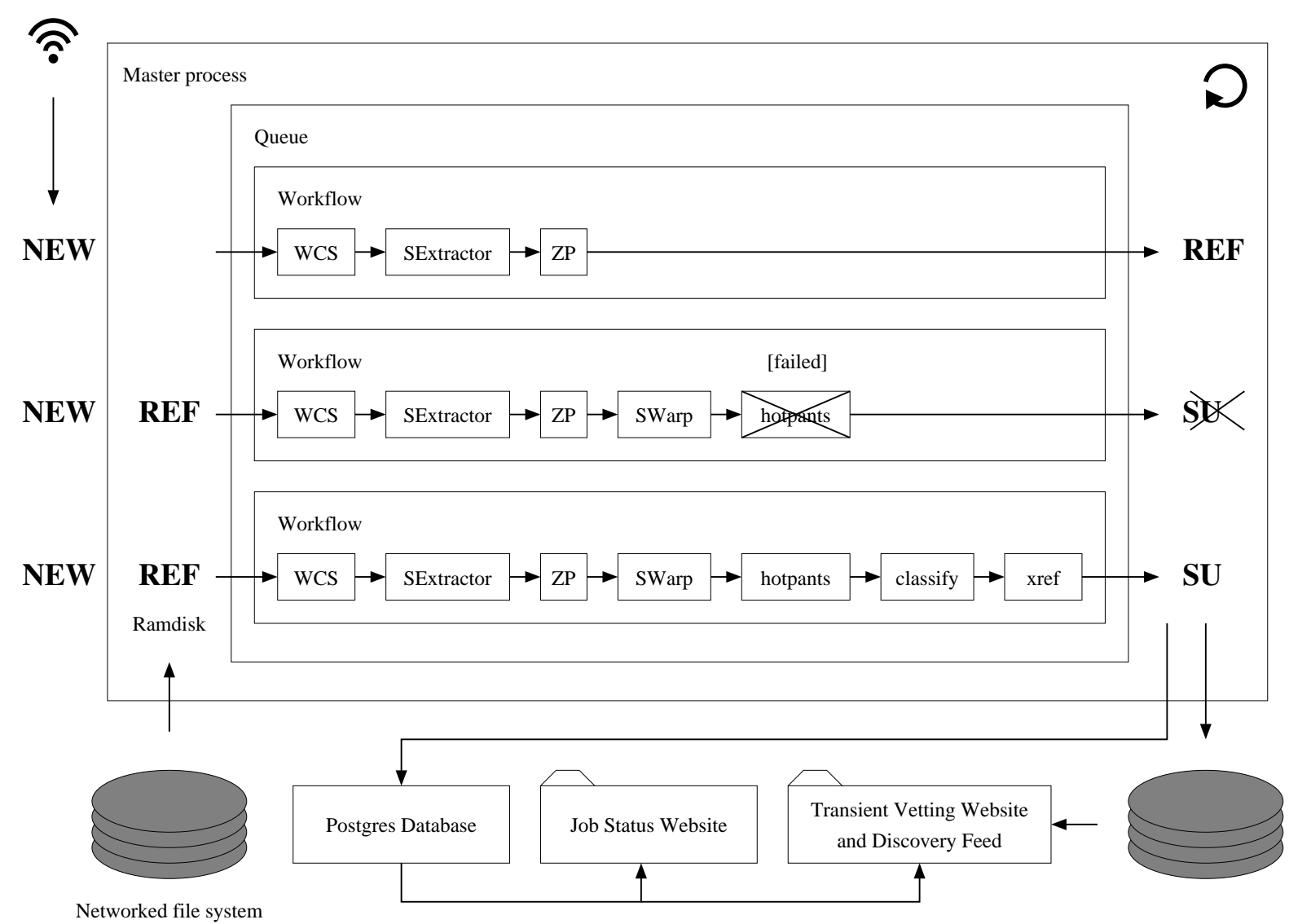

Figure 1. Architecture of the SkyMapper SN Search pipeline.

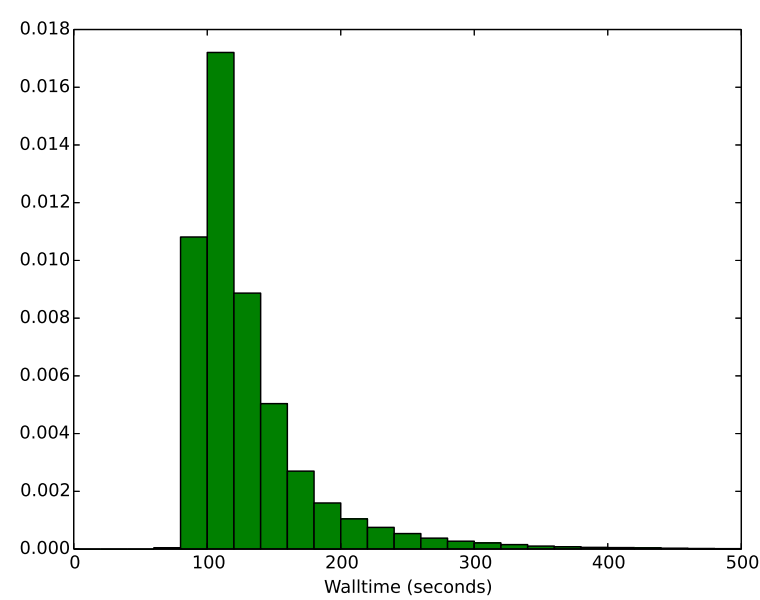

Figure 2. Distribution of processing times for successful subtraction jobs.

\subsection{Image subtraction pipeline job control}

The pipeline runs on a custom-built cluster named Maipenra 3 hosted at the Australian National University's Research School for Astronomy and Astrophysics (RSAA) in Canberra. The cluster has 48 cores with 192 GB of random-access memory and $44 \mathrm{~TB}$ of network-mounted disk space. A small part of available memory (32 GB) is set aside as a fast virtual file system ("ramdisk"). This ensures that I/O-intensive processing by third-party image processing programs can be performed directly in memory without modifying the code, dramatically improving performance. Relational information about images, pipeline jobs, and transient objects discovered is hosted in a Postgres database, accessed through the Django web framework 4 . Commonly used catalogs, such as UCAC2 (Zacharias et al.2000), 2MASS (Skrutskie et al. 2006), and APASS (Henden \& Munari 2014), are accessed via a separate Postgres database hosted locally at RSAA. Figure 1 also shows a schematic representation of the flow control for the pipeline. To produce an architecture that is efficient, fault-

${ }^{3}$ http://www.maipenrai.com.au

${ }^{4}$ https://djangoproject.com 
tolerant, and transparent, we adhere to the following design principles:

1. A master process coordinates assignment of jobs to up to 32 worker processes at a time, monitoring their state through a polling loop. Each SkyMapper exposure is reduced using one worker process per $2048 \times 4096$ $\mathrm{CCD}$, so that individual images fit easily in memory and no special software for reducing large mosaic images is needed.

2. Only the master process is allowed to transfer inputs and outputs of worker processes between shared disk and the ramdisk, which it does synchronously at the beginning and end of a polling cycle. This prevents many worker processes from accessing shared disk at once, placing minimal strain on networked file systems.

3. Although worker processes can query databases, the master process is responsible for updates to database tables, aggregating results from various processes to minimise I/O overhead.

4. The SN Survey layout is organised around a set of fixed fields on the sky, corresponding to a subset of the Main Survey fields. All shared disk storage is organised into subpaths corresponding to unique field/filter/CCD combinations.

5. Each worker process runs through a modular workflow, logging both the system calls needed to execute particular steps and the output of those steps to a log file. Any step which fails can be rerun easily based on the logged system calls, speeding up debugging. Process status and $\log$ files can be accessed quickly through a web interface.

\subsection{Selecting candidates for photometric follow-up}

The astrometric, photometric, and PSF matching of the REF to the NEW will in general not be perfect. Image subtraction artifacts not corresponding to astrophysical variable objects are easily recognizable to the human eye as anything in the SUB image not resembling a point source. However, these artifacts are much more numerous, outnumbering true astrophysical variable objects by more than an order of magnitude even in relatively clean subtractions. Initial triage of detected objects on subtractions must therefore be automated.

To address this challenge, we have implemented a series of machine learning classifiers to distinguish "Real" astrophysical objects from "Bogus" artifacts or cosmetic features appearing in the search images. Our first version of this classifier used the Python-based machine learning package MILK ${ }^{5}$ to implement a random forest classifier modeled after Bloom et al. (2012). For more recent versions, we have switched to the random forest implementation in SKLEARN (Pedregosa et al. 2011), which trains more quickly and makes cross-validation easier.

\footnotetext{
${ }^{5}$ https://github.com/luispedro/milk
}

At all stages of its development, the performance of the classifier has been limited mainly by the availability of training examples of Real SNe. The first version of the classifier used training data from early SkyMapper commissioning (August 2011), based on a sample of detections visually scanned and tagged as visually similar to Real or Bogus detections by human scanners. When evaluated against Real SN detections from contemporary data, this version of the classifier performs no better than random chance - possibly due to the lack of confirmed Real supernovae in the training set, and to the dramatic changes in the SkyMapper PSF from commissioning through to current operations. After the Zooniverse campaign in March 2015, we retrained the classifier on a larger sample of Real detections of supernovae discovered by the pipeline (see Table 1), supplemented by a random selection of asteroids of varying magnitude as examples of Real objects visually resembling (hostless) supernovae in single exposures. A third version was trained in October 2016 using Real discoveries from the first year of full-time operations. As the number of Real supernovae increased, successive retrainings have reduced our dependence on non-supernova detections tagged as Real, producing progressively more accurate results.

We evaluate the performance of all classifier models using $k$-fold cross-validation, in which the data are divided into $k$ disjoint subsets, with $k-1$ subsets reserved for training and the final subset used for validation. This technique enables most of the data to be used for training while determining the impact of certain subsets of data on classifier robustness. We chose $k=5$ for our training. To make a fair estimate of the generalization error from our small sample of Real supernovae, we placed multiple detections of the same supernova in the same fold. This ensures that the training accounts for variations in observing conditions and supernova magnitude, while the uncertainty in SN performance fairly reflects variations in host galaxy background and contrast, to which our classifier will be vulnerable when evaluating new detections. The total dataset for cross-validation includes 688 Real detections of 57 supernovae of all spectral types, 1351 Real detections of asteroids, and 4479 randomly selected Bogus detections; the

We evaluate the classifier's performance according to the efficiency (1.0 minus the missed detection rate) and purity (1.0 minus the false positive rate) of the classified candidates. Figure 3 shows the Receiver Operating Characteristic (ROC) curve of these measures against each other, averaged over folds, demonstrating the trade-off resulting by varying the score threshold separating the Real and Bogus classes. The more recent classifier version is more efficient at high purity, with about $70 \%$ efficiency at $99 \%$ purity (somewhat worse than the Bloom et al. (2012) classifier on which it is based). Figure 4 shows the efficiency of the more recent classifier version as a function of signal-to-noise ratio of the detection, demonstrating recent improvements in the effective signal-to-noise threshold and retention of bright detections. All versions of the classifier are archived and labelled so that 


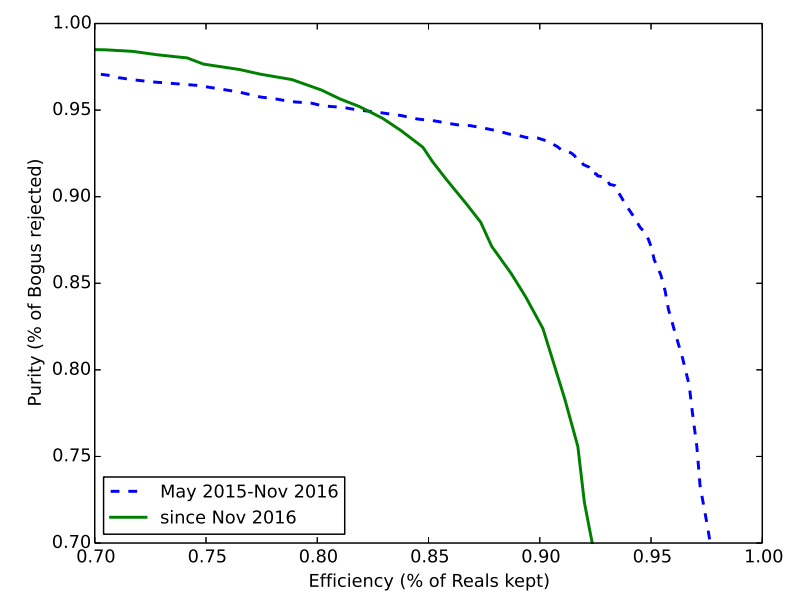

Figure 3. ROC curve (averaged among folds) for Real/Bogus classifier results.

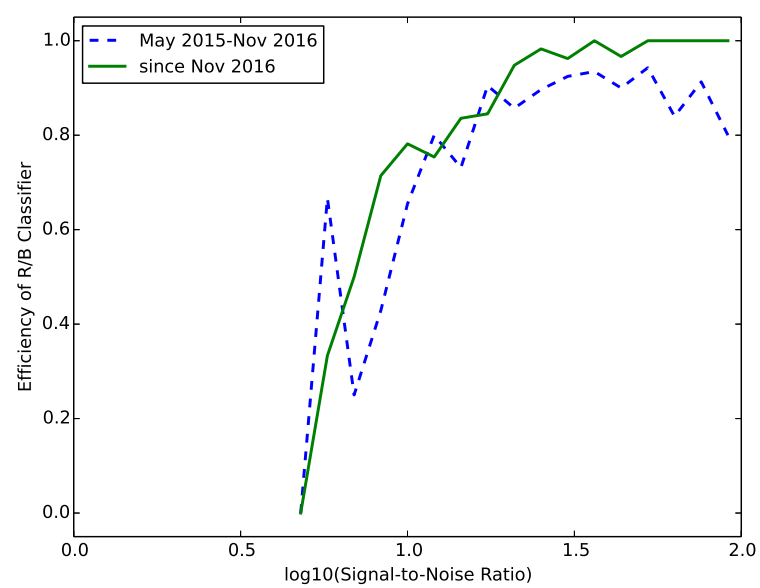

Figure 4. Classifier efficiency as a function of detection signal-to-noise.

the selection function for candidates can be reconstructed for later studies (e.g., SN rates or SN Ia cosmology).

To further reduce the rate of false positives, we also require at least two Real detections of an object at the same location on separate nights or filters. Objects passing this cut are astrometrically matched to existing catalogs, including APASS (Henden \& Munari 2014), the 2MASS Point Source Catalog (Skrutskie et al. 2006), and the SkyBot virtual observatory service for asteroids (Berthier et al. 2006). Any match better than 1 arcsec to the position of a known point source will cause the classification of that source to be carried over to the new candidate.

Events with at least two detections (in any band) are passed on with annotations to astronomers to be reviewed for potential follow-up after every night of observing. The historical light curve is available for review, showing photometric detections and upper magnitude limits throughout the recent history of observations of each field.

\subsection{Follow-up}

Once a candidate is selected for follow-up, it is placed in a queue for intensive monitoring by the SkyMapper telescope on a nominal 4-day cadence for gri (5-day for $v$ ), to ensure high-quality post-detection light curves.

Spectra for classification and scientific follow-up are taken as part of the ANU WiFeS SuperNovA Program (AWSNAP; submitted to PASA), using the WiFeS integral field spectrograph (Dopita et al. 2007) on the RSAA 2.3$\mathrm{m}$ telescope at Siding Spring Observatory, and as part of other spectroscopic surveys such as the Public ESO Spectroscopic Survey of Transient Objects (Smartt et al. 2014) and the Las Cumbres Observatory Global Telescope Network (Brown et al. 2013). Follow-up targets are shared with PESSTO via a live feed, and as of mid-2016 WiFeS observations take place in Target of Opportunity (ToO) mode. An API to report SMT transient candidates through the Transient Name Servert is already implemented and working since December 2016. Transients reported can be also found in the SMT webpage 7 .

\section{SURVEY STRATEGY AND SIMULATIONS}

Here we discuss the survey strategy for the SkyMapper SN Survey, which serves to maximise the number of wellsampled SN Ia light curves that can be included in a cosmology sample.

\subsection{Search and follow-up strategy}

The SkyMapper SN Survey strategy is tuned to discover SNe Ia in the local universe $(z<0.1)$, uniformly distributed in solid angle at high galactic latitudes $(b>30)$, and to produce high-quality multi-band light curves for cosmology. The strategy includes two components:

1. Rolling search mode: The telescope observes on a regular cadence ( $\leq 5$ days) in the SkyMapper $g r$ bandpasses. This mode does not explicitly target known galaxies, in order to produce a selection function as similar as possible to high-redshift SN Ia surveys such as SNLS.

2. Follow-up mode: The telescope follows up fields with active supernovae using a tighter cadence with griv bandpasses (see Section 3.4). Although follow-up does not require SkyMapper's wide field, it ensures that

\footnotetext{
${ }^{6}$ https://wis-tns.weizmann.ac.il/

${ }^{7}$ http://www.mso.anu.edu.au/skymapper/smt/
} 


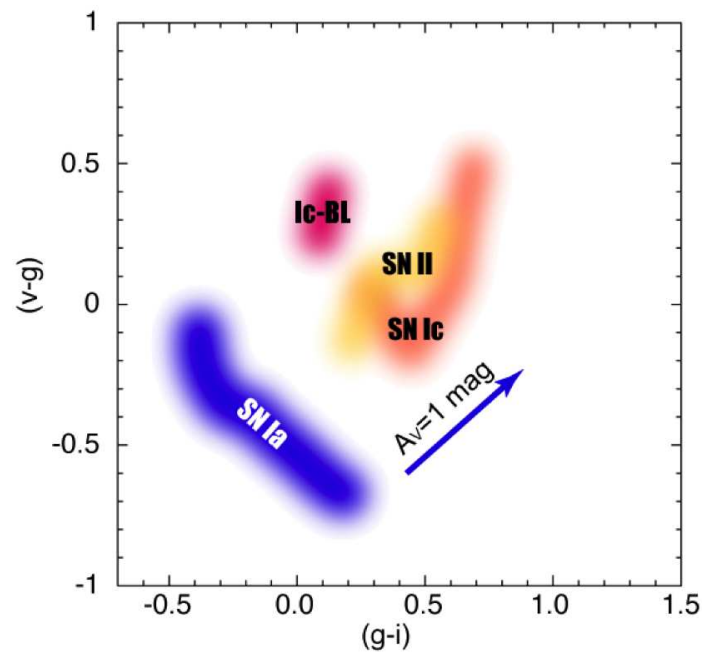

Figure 5. Subclasses of supernovae, including Type Ia and core-collapse (normal and broad-line Type Ic and Type II) in a $v-g-g-i$ colour-colour plot. This figures shows that $v$-band lightcurve points provide colour information for photometric selection of candidates to complement spectroscopic classification.

a uniform accurate calibration applies both to prediscovery photometry from the rolling search and to the follow-up photometry. This mode also enables the SN Survey to trigger on supernovae found in other public supernova searches, to boost statistics and enable cross-calibration of SkyMapper photometry with photometry from other groups.

The exposure times for each bandpass are constrained by the desired limiting magnitude of our survey of $\sim 20.5-21$. The chosen exposure times are $100 \mathrm{sec}$ in $g$ and $r, 300 \mathrm{sec}$ in $i$, and $500 \mathrm{sec}$ in $v$. Early SkyMapper $v$-band exposures are potentially valuable for photometric discrimination between $\mathrm{SNe}$ Ia and other types of supernovae (see Figure 5), and for examining the influence of progenitor metallicity on SN Ia luminosities. The $v$-band is read-noise dominated for exposures less than about $500 \mathrm{sec}$ long. We therefore include the triggered follow-up of $500 \mathrm{sec} v$-band observations after the discovery of each SN Ia in our total observing time budget.

SMT uses bad-seeing conditions which are less useful Main Survey imaging, as it involves only detection of bright point sources in relatively sparse fields. Our program uses the worst $25-30 \%$ of seeing conditions for observations in the transient search, corresponding to a threshold seeing of $>2.3^{\prime \prime}$. This is supplemented by time in any conditions (including good-seeing) to maintain a semi-regular cadence on active SN fields in follow-up mode.

The number of fields covered by the footprint is constrained by the desired cadence, depth, and wavelength coverage, as well as the total amount of telescope time available to the survey. To better understand these trade-offs, we are carrying out additional simulations which will take into ac- count the characteristics of the instrument (throughput, PSF, read-out noise sky background for each filter) and historical variations in weather conditions at Siding Spring Observatory.

\subsection{Survey geometry}

Coherent peculiar motions in the local Universe produce spatially correlated deviations in peculiar velocities from a uniform Hubble flow; accurate constraints on bulk flows require coverage over a large area on the celestial sphere (Hui \& Greene 2006). Unless the entire sky is covered uniformly, survey geometry may affect the final performance of the survey. Haugbølle et al. (2007) argued that accurate measurements of peculiar velocities required a survey geometry that minimised the size of holes in the footprint. The large dust extinction in the plane of the Galaxy constrains the survey geometry, since Milky Way dust is the second-largest source of systematic uncertainty in SN Ia distances (after photometric calibration) in contemporary SN Ia Hubble diagrams (Conley et al. 2011; Betoule et al. 2014).

The influence of different survey geometries on bulk flow constraints for the SkyMapper SN Survey has been simulated in Scrimgeour et al. (in prep) including random sets of fields selected uniformly in area, "glass" geometries meant to minimise holes in coverage, and geometries avoiding the Galactic plane (with a maximum Milky Way extinction or minimum Galactic latitude). They found that the total number of SNe Ia discovered and the combination of the SkyMapper SN Ia sample with northern-hemisphere samples (such as PTF) were each more influential factors than the choice of any specific survey geometry. Therefore, it makes sense for the SkyMapper SN survey to choose fields to minimise Galactic extinction, although new fields may be added as the survey progresses, in order to improve constraints on the bulk flow.

The SkyMapper SN Survey will therefore concentrate on a set of low-extinction fields $\left(E(B-V)_{\mathrm{MW}}<0.05\right)$. This strategy will ensure the pre-existence of deep galaxy references, which dramatically increase the expected SN Ia yield in our simulations (by nearly a factor of 2) relative to the Science Verification case where galaxy reference images are comparable in depth to the search images.

Additionally, we are following areas of the sky currently prioritised for Main Survey coverage by other extra-galactic programs, including the Shapley Supercluster and the footprint of the Kepler Extra-Galactic Survey (KEGS), which is using the Kepler K2 mission to monitor supernova fields at a very high-cadence of 30 minutes. K2 fields 1, 3, 4, 5, 6, $8,10,12,14,16$, and 17 have been KEGS focused fields, discovering to date 23 supernova, several of which been observed by SkyMapper (Rest et al. (in prep), Tucker et al. (in prep), Zenteno et al. (in prep)). 


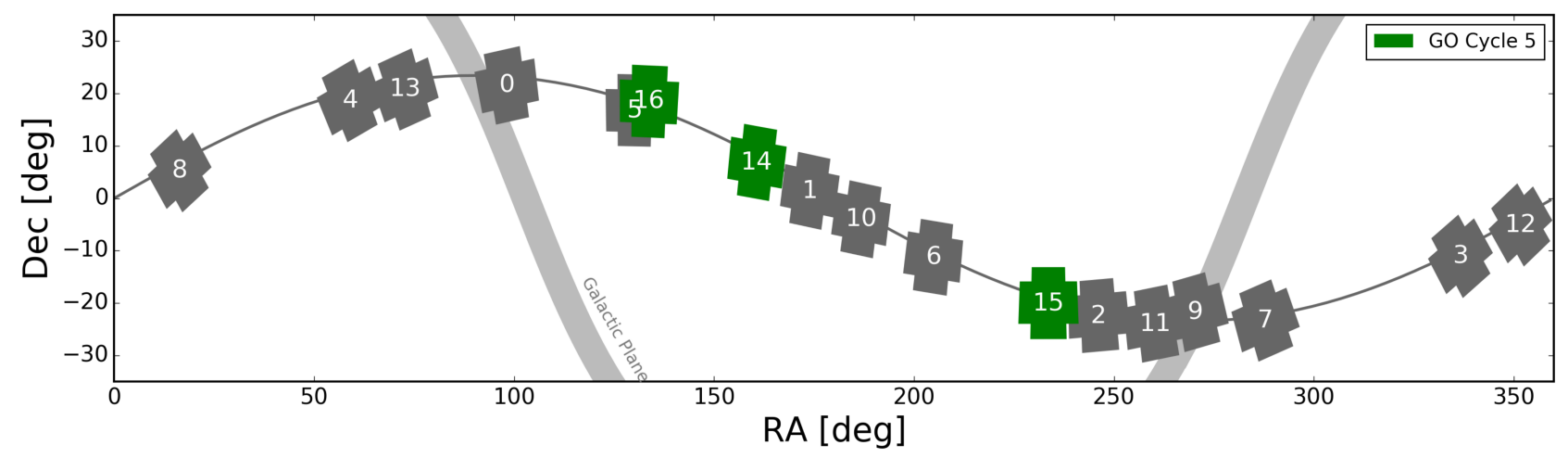

Figure 6. Figure courtesy of the NASA Kepler Guest Observer office. These are the footprints for the K2 campaigns, which lie along the ecliptic, with the green fields being observed in 2017. The Kepler Extra-Galactic Survey is monitoring, which SkyMapper is shadowing with ground-based multi-color observations, supernova in Campaigns 1, 3, 4, 5, 6, 8, 10, 12, 14, and 16.

\section{SURVEY PERFORMANCE}

In this section we summarise the data taken for the SkyMapper supernova survey, and evaluate its performance based on expectations from detailed simulations of the survey history.

\subsection{Early survey}

SkyMapper's performance has evolved over the commissioning period. Early SkyMapper images were limited in quality by vibrations at $\sim 30 \mathrm{~Hz}$ driven by a resonance with the cooling system for the camera; these were mitigated by modifications to change the telescope's resonant frequency. During the Science Verification period for the SN Survey (04 September 2013 to 09 March 2014), the median image quality was $\sim 3.5$ arcsec. After March 2014, additional improvements to focus and tracking resulted in a median image quality near 2 arcsec in gri bands. The readout overhead has also decreased, from a mean of 45 seconds during Science Verification to 21 seconds as of April 2014.

From April 2014 through April 2015, the SkyMapper telescope was dedicated to calibration of the SkyMapper standard star network (the Short Survey described in $\S 2$. This work involved observations of bright stars, taking many repeat images of the sky with short $(5-10 \mathrm{sec})$ exposure times and no planned cadence.

\subsection{Image quality}

Figure 7 shows the distribution of SkyMapper seeing in a representative filter $(g)$, in comparison to the distribution obtained from weather logs at the Anglo-Australian Telescope (AAT) assumed to be the natural seeing of the Siding Spring site. To assist us in running simulations to determine the performance of our survey over a long historical period using past weather logs, we developed a transfer function to pre- dict SkyMapper seeing from weather log entries. The model takes into account nightly variations in seeing (measured at the AAT), wavelength dependence, and airmass: for a filter with effective wavelength $\lambda$ and seeing $s_{\lambda}$,

$$
s_{\lambda}^{2}=s_{\mathrm{SM}, 0}^{2}+\left[s_{A A T}^{1+\alpha} X^{\gamma}\left(\frac{\lambda}{5500 \AA}\right)^{\beta}\right]^{2},
$$

where $s_{\mathrm{SM}, 0}=1.1 \operatorname{arcsec}$ is a baseline seeing floor, and $\alpha$, $\beta$, and $\gamma$ are coefficients characterizing atmospheric scattering. For contemporary data (taken after April 2014), chisquare minimization produces best-fit values $\alpha=-0.156$, $\beta=-0.5, \gamma=0.8$.

\subsection{Delays and limitations}

Through 2015 to early 2016 the SkyMapper telescope experienced various technical and software difficulties which delayed progress. These have typically halted survey operations for periods of 1-2 months at a time, during OctoberNovember 2015, and January and March 2016. Long periods of inclement weather (see Figure 8) during the winter have frustrated the search for SNe, with both SkyMapper and the $2.3 \mathrm{~m}$ telescope often being closed. This has adversely affected light curve quality and sampling, with numerous promising candidates fading before classification was possible, and/or left with large gaps in the light-curve.

As more of the Main Survey footprint (Figure 9 ) is completed, more SMT fields will have deep references. This is decrease the time cost of building REF images during SMT time, compared to the early survey. The search has operated continuously from April 2016, during which supernovae have steadily been discovered and classified. 

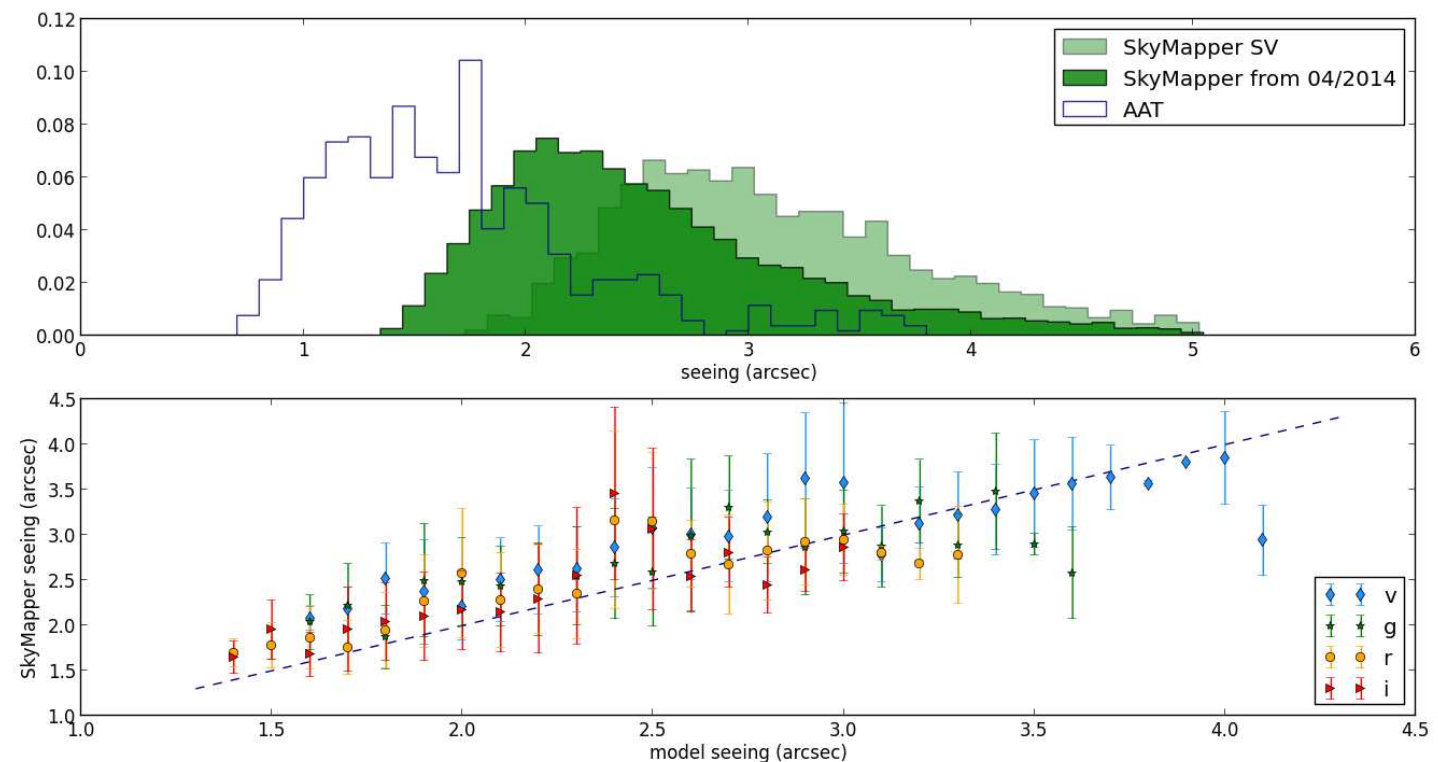

Figure 7. Top: Histograms of $g$-band seeing for the SkyMapper telescope during Science Verification (hatches) and after additional hardware intervention completed April 2014 (bold hatches), as compared to AAT seeing logs (open). Bottom: SkyMapper seeing in vgri bands from April 2014 - May 2015, compared with predictions from the transfer function.

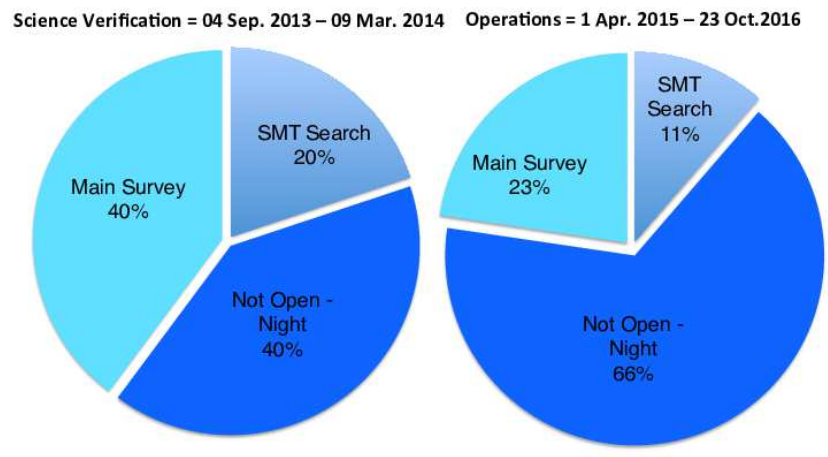

Figure 8. Impact of weather on Main Survey and Transient Survey operations as of 23 October 2016.

\section{FIRST RESULTS}

\subsection{First supernovae: Science Verification and Galaxy Zoo}

Table 1 reports the types and numbers of confirmed SNe discovered (see Figure 10 for color composite images) during early SkyMapper operations. During Science Verification, the Search produced 10 spectroscopically confirmed supernovae, among them 8 SNe Ia.

Supernova observations resumed during the period 12-20 March 2015, during which SkyMapper performed an intensive observing campaign with a short cadence of 1-2 days, as an outreach effort in partnership with the Galaxy Zoo citizen science community. An additional five spectroscopically confirmed $\mathrm{SNe}$ were discovered in this campaign, including three SNe Ia. Thumbnails of candidates found in the search appeared on the Galaxy Zoo website, and were processed by volunteers using the decision tree described in Smith et al. (2011). Popular candidates were inspected by the authors and submitted in real time for spectroscopic classification by the PESSTO collaboration using the ESO NTT 3.6-m telescope at La Silla. An example of typical light curve quality in the early survey is shown in Figure 11 for SMTJ10310056-3658262, a type Ia supernova discovered during the Galaxy Zoo campaign.

Figure 9 shows a map of the total supernova survey coverage to date. A total of 393 SkyMapper fields, or $2250 \mathrm{deg}^{2}$, have been observed, with a mean of 40 visits since the beginning of Science Verification. The expected number $N$ of supernovae to be found in the survey can be estimated by Monte Carlo, integrating

$$
N=\int d \Omega \int d t \int d z \frac{\partial V}{\partial z} \eta(z, \Omega, t)
$$



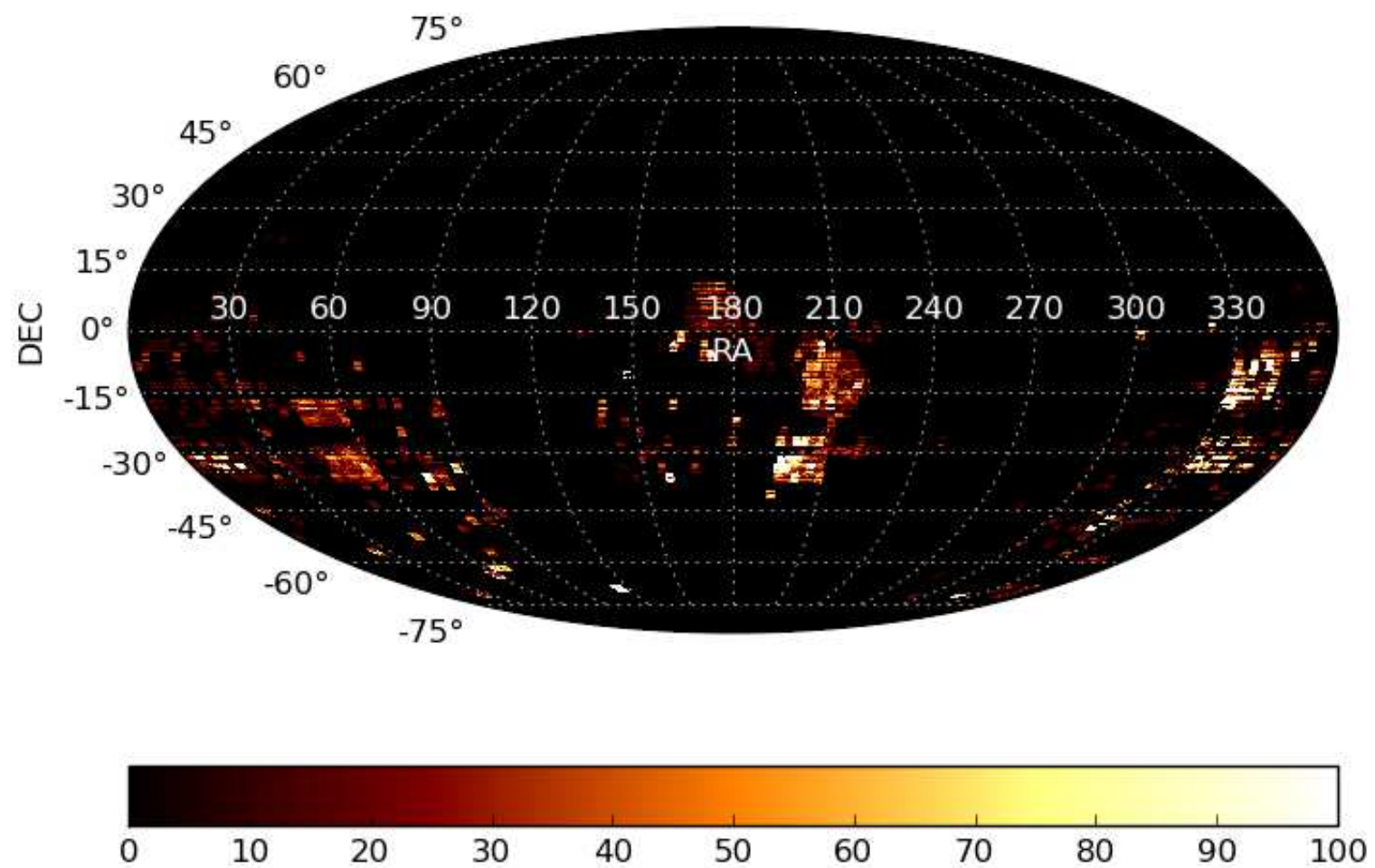

Figure 9. Map of cumulative sky coverage for the SkyMapper supernova search as of 20 October 2016.
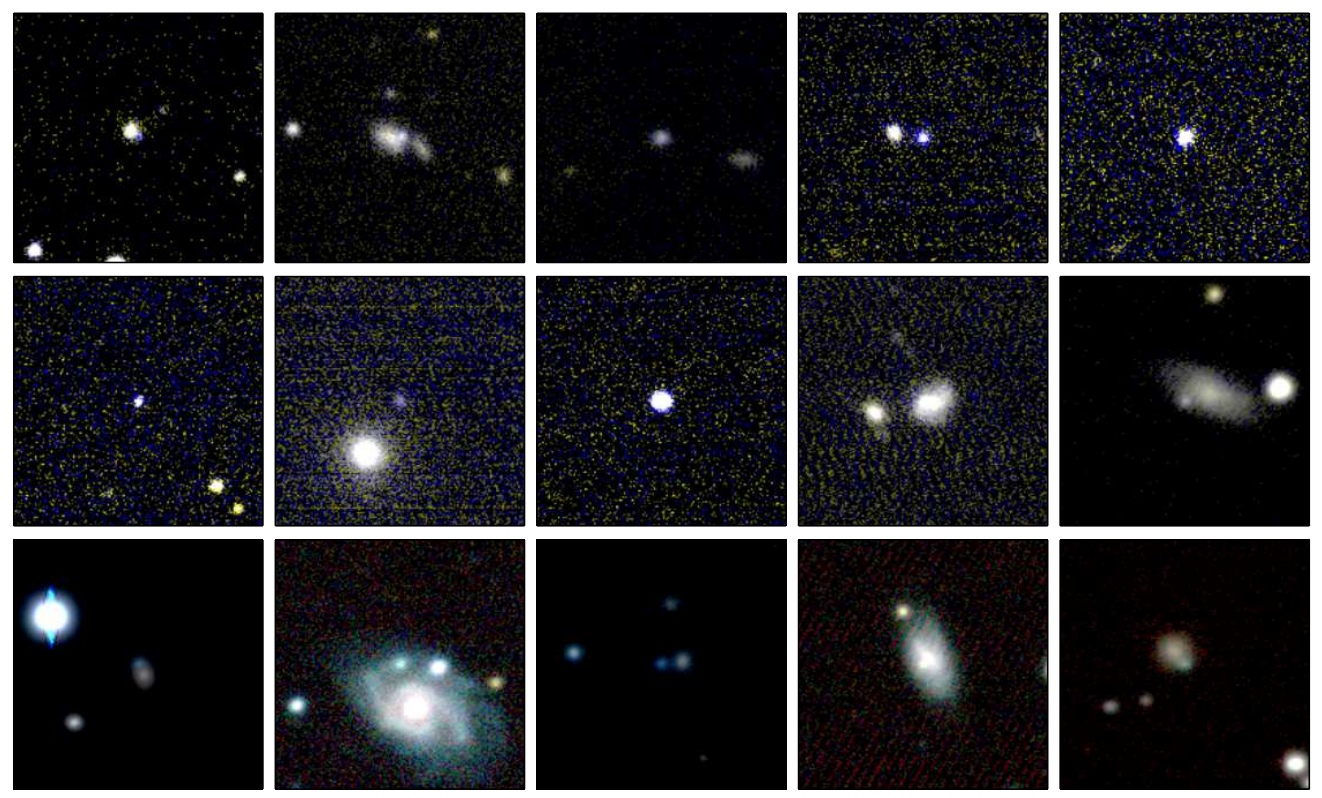

Figure 10. Color composite thumbnail images of a selection of early SkyMapper supernova discoveries. 
Table 1 Spectroscopically typed supernova discoveries during early SkyMapper operations

\begin{tabular}{|c|c|c|c|c|c|c|}
\hline Name & Disc. MJD (Phase) $)^{a}$ & RA & DEC & $z$ & Type & ATel \# \\
\hline \multicolumn{7}{|c|}{ Science Verification (04 Sep 2013 - 09 Mar 2014) } \\
\hline SMT J21413915-5643445 & $56573.6(-8)$ & $21: 41: 39.15$ & $-56: 43: 44.5$ & 0.142 & Ia & 5480 \\
\hline SMT J23032187-6911189 & $56592.6(-3)$ & 23:03:21.87 & $-69: 11: 18.9$ & 0.060 & Ia & $5521^{b}$ \\
\hline SMT J03054854-2850370 & 56626.6 & 03:05:48.54 & $-28: 50: 37.0$ & 0.050 & IIn & 5622 \\
\hline SMT J03253351-5344190 & $56627.6(-12)$ & $03: 25: 33.51$ & $-53: 44: 19.0$ & 0.055 & Ia & 5641 \\
\hline SMT J00570507-3626231 & $56628.8(+3)$ & 00:57:05.07 & $-36: 26: 23.1$ & 0.057 & Ia & 5620 \\
\hline SMT J03264288-3438055 & $56638.6(+14)$ & $03: 26: 42.88$ & $-34: 38: 05.5$ & 0.1 & Ia & $5602^{c}$ \\
\hline SMT J03101002-3637448 & $56653.6(+11)$ & 03:10:10.02 & $-36: 37: 44.8$ & 0.070 & Ia & $5650^{d}$ \\
\hline SN 2013hx & 56653.8 & $01: 35: 32.83$ & $-57: 57: 50.6$ & 0.130 & SLSN & 5912 \\
\hline SMT J04043173-6350154 & $56664.5(+2)$ & 04:04:31.73 & $-63: 50: 15.4$ & 0.1 & Ia & $5748^{e}$ \\
\hline SMT J05451320-4735425 & $56666.7(-5)$ & $05: 45: 13.20$ & $-47: 35: 42.5$ & 0.050 & Ia & - \\
\hline \multicolumn{7}{|c|}{ Zooniverse Campaign (12-22 Mar 2015) } \\
\hline SMT J10310056-3658262 & $57094.5(+0)$ & $10: 31: 00.56$ & $-36: 58: 26.2$ & 0.035 & Ia & 7261 \\
\hline SMT J13254308-2932269 & 57094.6 & $13: 25: 43.08$ & $-29: 32: 26.9$ & 0.040 & Ic & 7254 \\
\hline SMT J13545988-2820020 & $57094.6(+0)$ & $13: 54: 59.88$ & $-28: 20: 02.0$ & 0.038 & Ia & 7261 \\
\hline SMT J14323134-1339275 & 57095.7 & $14: 32: 31.34$ & $-13: 39: 27.5$ & 0.021 & IIb & $7261^{f}$ \\
\hline SMT J13481313-3325189 & $57094.6(+21)$ & $13: 48: 13.13$ & $-33: 25: 18.9$ & & Ia & $\simeq^{g}$ \\
\hline
\end{tabular}

${ }^{a}$ Phase in days relative to $B$-band maximum light (type Ia only).

${ }^{b}$ Discovered independently and first confirmed as PSNJ23032177-6911185 by the CHASE survey.

${ }^{c}$ Discovered independently and first confirmed as LSQ13dby.

${ }^{d}$ Discovered independently and first confirmed as LSQ13dkp.

${ }^{e}$ Discovered independently as OGLE-2014-SN-002.

${ }^{f}$ Discovered independently as LSQ15rw.

${ }^{g}$ Classified by LCOGT as a Ia well after maximum light (G. Hosseinzadeh, priv. comm).

over time $t$, the survey footprint $\Omega$, and redshift $z$; here $d V$ is the co-moving volume element at redshift $z$, and $\eta$ is the survey efficiency for discovery of supernovae including the particulars of the timing and depth of each image relative to a randomly generated set of supernova light curves. We estimate an effective significance threshold of $9 \sigma$ for singleepoch detections based on the empirical Real/Bogus classifier efficiency curve (Figure 4). We use the historical cadences and upper limits from successful subtractions.

Based on these assumptions, in the period AprilNovember 2016, when the instrument configuration was stable and the survey was working well, we expect a total of $65 \pm 8 \mathrm{SNe}$ Ia to be found by the survey in that period, assuming 2 significant detections on separate nights in any filter were necessary for detection. If instead simultaneous detections in both $g$ and $r$ are required, we expect $41 \pm 6$ SNe Ia instead. Our total of 13 spectroscopically confirmed SNe Ia during this period is lower than expected, with a number of possible factors contributing: losses in the classifier at the pixel level (not simulated at this stage), departures of the SkyMapper PSF from the assumed (round Gaussian) PSF up through Science Verification, edge effects due to slight pointing differences between NEW and REF, and spectroscopic selection. Of these effects, we expect that selection for spectroscopic follow-up is probably the largest of these effects, due to weather-induced gaps in light curve cover-

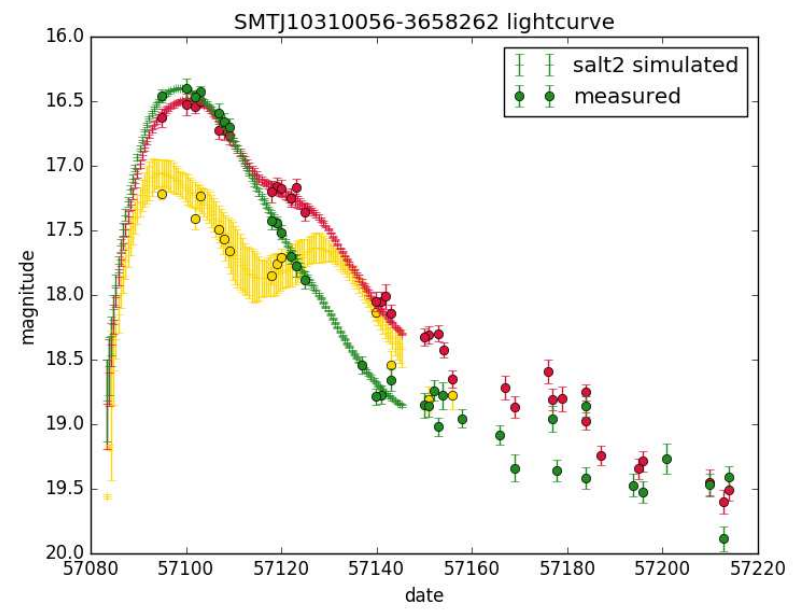

Figure 11. Lightcurve in gri colours of SMTJ10310056-3658262 discovered during the Galaxy Zoo campaign, shown with SALT2 fit.

age and follow-up availability. We identify an additional 39 transient objects found in our survey in 2016 with appropriate light curve timescales and visible host galaxies, but no spectra. 


\subsection{Unique peculiar objects}

The SkyMapper Transient Search is also discovering other types of supernova and stellar transients, such as superluminous supernovae Quimby et al. 2011; Gal-Yam 2012) The first notable SkyMapper object was indeed one of these, SN 2013hx (discovered as SMT J013533283-5757506), a superluminous supernova initially similar to SN 2010gx (Pastorello et al. 2010) but displaying broad $\mathrm{H} \alpha$ emission at late times (Inserra et al. 2016). SN 2013hx was discovered by SkyMapper at MJD 56657.6 (2013 Dec 31 UT), and reached reached peak magnitudes $g=16.9, r=17.0$ at MJD 56683.5 (2014 Jan 26 UT). At a redshift of 0.130, SN 2013hx was the closest superluminous type II supernova discovered to date (Nicholl et al. 2014), presenting an excellent opportunity for late-time observations. SN 2013hx was also included in a recent study of superluminous supernova light curves (Nicholl et al. 2015).

We are also sensitive to more exotic transients, such as faint calcium-rich transients (Kasliwal et al. 2012), which occur preferentially in low-surface-brightness, star-forming host galaxies (Neill et al. 2011) or on the outskirts of larger galaxies not monitored by targeted searches. Yuan et al. 2013) We have already discovered two exotic transients: SN 2015J, a possible SN impostor or magnetar-powered SN Ic (Tucker et al. in prep), and an object similar to Arcavi et al. (2016) in the so-called superluminous-gap (Zhang et al. in prep). We may also be sensitive to shock interaction with SN Ia companions, as observed in SN 2016hhd. (Möller et al. in prep)

Moreover, we are also triggering on other exotic multiwavelength events such as Fast Radio Bursts (FRBs) and Gravitational Wave (GW) alerts. Additionally, the data should also be useful for studying variable stars, active galactic nuclei, and other types of transients, and can be coadded to provide deep exposures of the search area.

\section{SUMMARY}

This work presents the SkyMapper Transient Survey, including the software specific to the Transient Search and the planned survey strategy. The former involves a sophisticated image subtraction pipeline with a machine learning classifier and a web admin interface for human input. We describe the early performance of the Survey, which is steadily assembling a small sample of SNe Ia for inclusion in a lowredshift cosmology sample (to be detailed in a forthcoming data release paper), and in addition discovering peculiar objects interesting in their own right (Tucker et al., Zhang et al., Möller et al.; all in preparation).

\section{ACKNOWLEDGEMENTS}

Parts of this research were conducted by the Australian Research Council Centre of Excellence for All-sky Astrophysics (CAASTRO), through project number CE110001020. RS acknowledges support from ARC Laureate Grant FL0992131. This research was made possible through the use of the AAVSO Photometric Allsky Survey (APASS), funded by the Robert Martin Ayers Sciences Fund. We gratefully acknowledge the assistance of over 40,000 online volunteers for the Snapshot Supernova program in March 2015, run in partnership with the Zooniverse citizen science portal. We also thank Andy Howell and the LCOGT team for providing us with a spectroscopic classification for SMT J13481313-3325189. We acknowledge and thank PhD students: F. Panther, R. RiddenHarper and N.E. Sommer as well contributions by summer students: P. Armstrong, G. Taylor, E. Moore, C. Bray and Y. Chen.

\section{REFERENCES}

Arcavi, I., Wolf, W. M., Howell, D. A., et al. 2016, ApJ, 819, 35

Baltay, C., Rabinowitz, D., Hadjiyska, E., et al. 2013, PASP, 125,683

Berthier, J., Vachier, F., Thuillot, W., et al. 2006, in Astronomical Society of the Pacific Conference Series, Vol. 351, Astronomical Data Analysis Software and Systems XV, ed. C. Gabriel, C. Arviset, D. Ponz, \& S. Enrique, 367-+

Bertin, E., \& Arnouts, S. 1996, A\&AS, 117, 393

Bertin, E., Mellier, Y., Radovich, M., et al. 2002, in Astronomical Society of the Pacific Conference Series, Vol. 281, Astronomical Data Analysis Software and Systems XI, ed. D. A. Bohlender, D. Durand, \& T. H. Handley, 228

Bessell, M., Bloxham, G., Schmidt, B., et al. 2011, PASP, 123, 789

Betoule, M., Marriner, J., Regnault, N., et al. 2013, A\&A, 552, A124

Betoule, M., Kessler, R., Guy, J., et al. 2014, A\&A, 568, A22

Bloom, J. S., Richards, J. W., Nugent, P. E., et al. 2012, PASP, 124, 1175

Brown, T. M., Baliber, N., Bianco, F. B., et al. 2013, PASP, 125, 1031

Burns, C. R., Stritzinger, M., Phillips, M. M., et al. 2014, ApJ, 789, 32

Childress, M., Aldering, G., Antilogus, P., et al. 2013, ApJ, 770,108

Conley, A., Guy, J., Sullivan, M., et al. 2011, ApJS, 192, 1

Contreras, C., Hamuy, M., Phillips, M. M., et al. 2010, AJ, 139, 519

Dark Energy Survey Collaboration, Abbott, T., Abdalla, F. B., et al. 2016, MNRAS, 460, 1270

Davis, T. M., Hui, L., Frieman, J. A., et al. 2011, ApJ, 741, 67

Djorgovski, S. G., Baltay, C., Mahabal, A. A., et al. 2008, Astronomische Nachrichten, 329, 263

Dopita, M., Hart, J., McGregor, P., et al. 2007, Ap\&SS, 310, 255

Drake, A. J., Djorgovski, S. G., Mahabal, A., et al. 2009, 
ApJ, 696, 870

Filippenko, A. V., Li, W. D., Treffers, R. R., \& Modjaz, M. 2001, in Astronomical Society of the Pacific Conference Series, Vol. 246, IAU Colloq. 183: Small Telescope Astronomy on Global Scales, ed. B. Paczynski, W.-P. Chen, \& C. Lemme, 121

Gal-Yam, A. 2012, Science, 337, 927

Haugbølle, T., Hannestad, S., Thomsen, B., et al. 2007, ApJ, 661, 650

Henden, A., \& Munari, U. 2014, Contributions of the Astronomical Observatory Skalnate Pleso, 43, 518

Hicken, M., Challis, P., Jha, S., et al. 2009, ApJ, 700, 331

Hicken, M., Challis, P., Kirshner, R. P., et al. 2012, ApJS, 200, 12

Howell, D. A., Sullivan, M., Conley, A., \& Carlberg, R. 2007, ApJL, 667, L37

Hui, L., \& Greene, P. B. 2006, Phys. Rev. D, 73, 123526

Inserra, C., Smartt, S. J., Gall, E. E. E., et al. 2016, ArXiv e-prints, arXiv:1604.01226

Jha, S., Kirshner, R. P., Challis, P., et al. 2006, AJ, 131, 527

Kaiser, N., Burgett, W., Chambers, K., et al. 2010, in Society of Photo-Optical Instrumentation Engineers (SPIE) Conference Series, Vol. 7733, Society of Photo-Optical Instrumentation Engineers (SPIE) Conference Series, 0

Kasliwal, M. M., Kulkarni, S. R., Gal-Yam, A., et al. 2012, ApJ, 755, 161

Keller, S. C., Schmidt, B. P., Bessell, M. S., et al. 2007, PASA, 24, 1

Keller, S. C., Bessell, M. S., Frebel, A., et al. 2014, Nature, 506, 463

Kelly, P. L., Filippenko, A. V., Burke, D. L., et al. 2015, Science, 347, 1459

Kelly, P. L., Hicken, M., Burke, D. L., Mandel, K. S., \& Kirshner, R. P. 2010, ApJ, 715, 743

Kim, A. G., Linder, E. V., Miquel, R., \& Mostek, N. 2004, MNRAS, 347, 909

Law, N. M., Kulkarni, S. R., Dekany, R. G., et al. 2009, PASP, 121, 1395

Li, W. D., Filippenko, A. V., Treffers, R. R., et al. 2000, in American Institute of Physics Conference Series, Vol. 522, American Institute of Physics Conference Series, ed. S. S. Holt \& W. W. Zhang, 103-106

Milne, P. A., Foley, R. J., Brown, P. J., \& Narayan, G. 2015, ApJ, 803, 20

Neill, J. D., Sullivan, M., Gal-Yam, A., et al. 2011, ApJ, 727, 15

Nicholl, M., Smartt, S. J., Jerkstrand, A., et al. 2014, MNRAS, 444, 2096

—. 2015, ArXiv e-prints, arXiv:1503.03310

Pastorello, A., Smartt, S. J., Botticella, M. T., et al. 2010, ApJL, 724, L16

Pedregosa, F., Varoquaux, G., Gramfort, A., et al. 2011, Journal of Machine Learning Research, 12, 2825
Pel, J. W., \& Lub, J. 2007, in Astronomical Society of the Pacific Conference Series, Vol. 364, The Future of Photometric, Spectrophotometric and Polarimetric Standardization, ed. C. Sterken, 63

Perlmutter, S., Aldering, G., Goldhaber, G., et al. 1999, ApJ, 517,565

Phillips, M. M., Simon, J. D., Morrell, N., et al. 2013, ApJ, 779, 38

Quimby, R., Höflich, P., \& Wheeler, J. C. 2007, ApJ, 666, 1083

Quimby, R. M., Kulkarni, S. R., Kasliwal, M. M., et al. 2011, Nature, 474, 487

Rau, A., Kulkarni, S. R., Law, N. M., et al. 2009, PASP, 121, 1334

Regnault, N., Barrelet, E., Guyonnet, A., et al. 2012, ArXiv e-prints, arXiv:1208.6301

Riess, A. G., Filippenko, A. V., Challis, P., et al. 1998, AJ, 116, 1009

Riess, A. G., Kirshner, R. P., Schmidt, B. P., et al. 1999, AJ, 117,707

Schmidt, B. P., Suntzeff, N. B., Phillips, M. M., et al. 1998, ApJ, 507, 46

Scolnic, D. M., Riess, A. G., Foley, R. J., et al. 2014, ApJ, 780, 37

Skrutskie, M. F., Cutri, R. M., Stiening, R., et al. 2006, AJ, 131,1163

Smartt, S. J., Valenti, S., Fraser, M., et al. 2014, ArXiv eprints, arXiv:1411.0299

Smith, A. M., Lynn, S., Sullivan, M., et al. 2011, MNRAS, 412, 1309

Stubbs, C. W., Slater, S. K., Brown, Y. J., et al. 2007, in Astronomical Society of the Pacific Conference Series, Vol. 364, The Future of Photometric, Spectrophotometric and Polarimetric Standardization, ed. C. Sterken, 373

Sullivan, M., Ellis, R. S., Howell, D. A., et al. 2009, ApJL, 693, L76

Sullivan, M., Conley, A., Howell, D. A., et al. 2010, MNRAS, 406, 782

Sullivan, M., Guy, J., Conley, A., et al. 2011, ApJ, 737, 102

Wang, X., Filippenko, A. V., Ganeshalingam, M., et al. 2009, ApJL, 699, L139

York, D. G., Adelman, J., Anderson, Jr., J. E., et al. 2000, AJ, 120, 1579

Yuan, F., Kobayashi, C., Schmidt, B. P., et al. 2013, MNRAS, 432, 1680

Zacharias, N., Urban, S. E., Zacharias, M. I., et al. 2000, AJ, 120,2131 